CLNS-96/1402

hep-th/9602118

February 1996

\title{
Heterotic-Heterotic String Duality and Multiple K3 Fibrations
}

\author{
Paul S. Aspinwall \\ F.R. Newman Lab. of Nuclear Studies, \\ Cornell University, \\ Ithaca, NY 14853 \\ Mark Gross \\ Department of Mathematics, \\ Cornell University, \\ Ithaca, NY 14853
}

\begin{abstract}
A type IIA string compactified on a Calabi-Yau manifold which admits a K3 fibration is believed to be equivalent to a heterotic string in four dimensions. We study cases where a Calabi-Yau manifold can have more than one such fibration leading to equivalences between perturbatively inequivalent heterotic strings. This allows an analysis of an example in six dimensions due to Duff, Minasian and Witten and enables us to go some way to prove a conjecture by Kachru and Vafa. The interplay between gauge groups which arise perturbatively and nonperturbatively is seen clearly in this example. As an extreme case we discuss a Calabi-Yau manifold which admits an infinite number of $\mathrm{K} 3$ fibrations leading to infinite set of equivalent heterotic strings.
\end{abstract}




\section{Introduction}

It is now generally believed that the type IIA string compactified down to six dimensions on a K3 surface gives the same physics as a heterotic string compactified on a four-torus (see, for example, [ 1, 2]). It was suggested in [ 3, 4] that much the same effect could be seen in four dimensions for $N=2$ theories. That is, a type II string compactified on a Calabi-Yau threefold could be equivalent to a heterotic string compactified on an object, essentially a K3 surface times a two-torus, for a suitable Calabi-Yau manifold.

Let us suppose that we have a type IIA string compactified on a Calabi-Yau manifold, $X$. It was realized in [5] that some of the evidence supporting the conjectured dual pairs of [3] appeared to originate in the fact that $X$ could be written in the form of a K3 fibration. That is $X$ can be written as a bundle where the generic fibre is a K3 surface and the base space is $\mathbb{P}^{1}$. Over a finite number of points on the base $\mathbb{P}^{1}$ the fibre degenerates and will not be a K3 surface. It was realized in [ 6] that this fibration structure fits in nicely with the six-dimensional duality above.

A general result concerning K3 fibrations was derived in [7]. That is, if there is a heterotic string dual to the type IIA string compactified on $X$, such that the weakly-coupled heterotic string can be understood in terms of $X$ near its large-radius limit, then $X$ must be a K3 fibration. In particular, the divisor class of the K3 fibre (or equivalently, the size of the base $\mathbb{P}^{1}$ ) on the type IIA side maps to the dilaton on the heterotic side.

It is natural to ask whether the converse to this statement is true. That is, if $X$ is a K3 fibration then must there exist a heterotic dual? Certainly there is a field in the theory which has every right to be called a dilaton for the heterotic string. This question is analogous to one familiar from the relationship between conformal field theories and non-linear $\sigma$-models with a Calabi-Yau target space. When does a conformal field theory have a Calabi-Yau target space interpretation? A necessary condition for this is that the moduli space should have a large-radius limit point, that is a point of maximal unipotency [8]. It seems reasonable to say that this is also a sufficient condition - certainly no counter-example is known. This condition of maximal unipotency appears to be on the same footing as the K3 fibration condition above and so again it seems very reasonable to assert that it is again a sufficient condition.

This raises an immediate question. Suppose $X$ is such that it admits more than one K3 fibration. That is, I can fibre $X$ in two (or more) ways such that the resulting K3 fibres in each case are not homologous. We now have two (or more) heterotic strings, each of which can equally be called the dual of the type IIA theory. Since the K3 fibres are not homologous, the dilatons for the heterotic strings must correspond to different directions in the moduli space of vector moduli. Thus the relationship between the heterotic strings will not be manifest from the perturbative analysis of the heterotic string using conformal field theory. Thus, Calabi-Yau manifolds with multiple K3 fibrations give examples of nontrivial heterotic dual pairs in four dimensions. 
An example of dual heterotic strings in six dimensions was given recently by Duff, Minasian and Witten [9]. Each member of this pair gives an $N=1$ theory in six dimensions and so can be compactified on a two-torus to give $N=2$ in four dimensions. The resulting theory is precisely one discussed in [3]. Thus, if the conjectures of [3] are to be correct then the $X$ given as the dual partner must admit at least two fibrations. We will see that this is correct, aside from some minor subtleties, in section 2. We will also see this double fibration structure essentially requires $X$ to be unique.

An important part of the analysis of [9] was the necessity of the possible appearance of gauge groups which could not be understood perturbatively from the heterotic string. Such groups were discussed in the context of type IIA strings in [ []. We will see in section 3 that the dual fibration picture discussed in section 2 fits in perfectly with this picture. We will also see that the type IIA string predicts more than can be seen from simple analysis of either of the dual heterotic strings.

There is actually no limit to how many K3 fibrations some Calabi-Yau manifolds may admit. In section 4 we discuss an extreme case of an infinite number.

While preparing this paper we became aware of [10] which has some overlap with this work.

\section{A Double Fibration}

In this section we will discuss the example of [9, 3] in the context of a double fibration.

Whereas a type II compactification is specified by the space on which it is compactified, a heterotic string requires, in addition, a vector bundle structure in which the gauge fields of the heterotic string live. The heterotic strings in six dimensions studied in [9] were compactified on a K3 surface with the following vector bundle. Let $E$ be a stable $S U(2)$ bundle on a $\mathrm{K} 3$ with $c_{2}(E)=12$. Embed this instanton in an $E_{8}$-bundle. The $E_{8} \times E_{8}$ bundle required is simply the sum of two such bundles.

Since the structure group of this bundle commutes with an $E_{7} \times E_{7}$ subgroup, we expect to see an $E_{7} \times E_{7}$ gauge group in the resultant six-dimensional physics. This bundle may be deformed however, that is, there are massless scalars in the six-dimensional theory. Giving expectation values to these scalars will deform the bundle and "Higgs" part of the gauge group away. A generic point in the moduli space will have no gauge group whatsoever.

The analysis of [9] indicated that for any such heterotic string one can find a dual partner. The dual partner is physically the same as the original but the field identified as the dilaton is different - a strongly coupled string maps to a weakly coupled partner — and the other moduli determining the shape of the K3 surface and the moduli of the bundle undergo a nontrivial transformation. Phrased differently, the complete moduli space of these heterotic strings, including the dilaton, admits a nontrivial $\mathbb{Z}_{2}$ isometry which should be modded out by in order to obtain the true moduli space of inequivalent theories in six dimensions. 
Now let us compactify this heterotic string on $T^{2}$. The resulting theory then has a $U(1)^{4}$ gauge group - two $U(1)$ 's from the metric from the isometries of the torus and two from reduction of the $B$-field. This is one of the examples studied in [3]. One of the $U(1)$ 's comes from the $N=2$ supergravity multiplet in four dimensions and the other three come from vector multiplets. The vector bundle $E$ described above lives in a moduli space with 448 real dimensions. Thus, together with the 80 real dimensions of the moduli space of strings on a K3 surface, our original theory in six dimensions (without the dilaton) lived in a moduli space of $448+448+80=976$ dimensions. This gives the moduli space coming from the hypermultiplets in the four-dimensional theory. This space has a quaternionic structure there are $976 / 4=244$ hypermultiplets.

For this four dimensional theory to be dual to a type IIA string compactified on a CalabiYau manifold $X$ we therefore require $h^{1,1}(X)=3$ and $h^{2,1}(X)=243$ (the dilaton of the type II string gives one hypermultiplet). In [ [3] it was conjectured that $X$ is given by the blow-up of a hypersurface in a weighted projective space - namely the hypersurface given by

$$
x_{1}^{24}+x_{2}^{24}+x_{3}^{12}+x_{4}^{3}+x_{5}^{2}=0,
$$

in the space $\mathbb{P}_{\{1,1,2,8,12\}}^{4}$.

When this is blown-up, it is a K3 fibration as discussed in [5]. This then satisfies the condition from [7] that it be dual to a heterotic string. From the result of [9] however, this is not enough - we need it to be dual to two heterotic strings. Thus there must be two K3 fibrations of $X$. At first sight we are in trouble - the blow-up of the hypersurface defined by (11) does not admit two fibrations.

All is not lost however. The manifold $X$ has 243 deformations of complex structure. The equation (四) only has 242 deformations. Therefore there is one deformation of $X$ which takes it outside the class of those embedded in $\mathbb{P}_{\{1,1,2,8,12\}}^{4}$. When we deform outside this class, the fibration structure of $X$ changes. ${ }^{1}$

The result is that, at a generic complex structure, $X$ is not only a K3 fibration but can also be written as an elliptic fibration with base space $\mathbb{P}^{1} \times \mathbb{P}^{1}$. Fix a point in the first $\mathbb{P}^{1}$ factor. Then the fibration over the second $\mathbb{P}^{1}$ defines a $\mathrm{K} 3$ surface as an elliptic fibration.

1 The best way to see this is as follows: The projection $\mathbb{P}_{\{1,1,2,8,12\}}^{4} \rightarrow \mathbb{P}_{\{1,1,2\}}^{2}$ onto the first three coordinates induces an elliptic fibration of $X$ onto $\mathbb{P}_{\{1,1,2\}}^{2}$. The space $\mathbb{P}_{\{1,1,2\}}^{2}$ is singular and should be blown-up to give the "Hirzebruch surface" $F_{2}$. This elliptic fibration is easily seen to have a section, and hence is birationally equivalent to a Weierstrass model

$$
y^{2}=x^{3}+a x+b,
$$

where $a \in \Gamma\left(\omega_{F_{2}}^{-4}\right)$ and $b \in \Gamma\left(\omega_{F_{2}}^{-6}\right)$. (See [11] and also [12].) Now we can deform $F_{2}$ to $\mathbb{P}^{1} \times \mathbb{P}^{1}$, and deform the above Weierstrass model to

$$
y^{2}=x^{3}+a x+b,
$$

where $a \in \Gamma\left(\omega_{\mathbb{P}^{1} \times \mathbb{P}^{1}}^{-4}\right)$ and $b \in \Gamma\left(\omega_{\mathbb{P}^{1} \times \mathbb{P}^{1}}^{-6}\right)$. This yields the additional deformation of complex structure not visible in $\mathbb{P}_{\{1,1,2,8,12\}}^{4}$. 


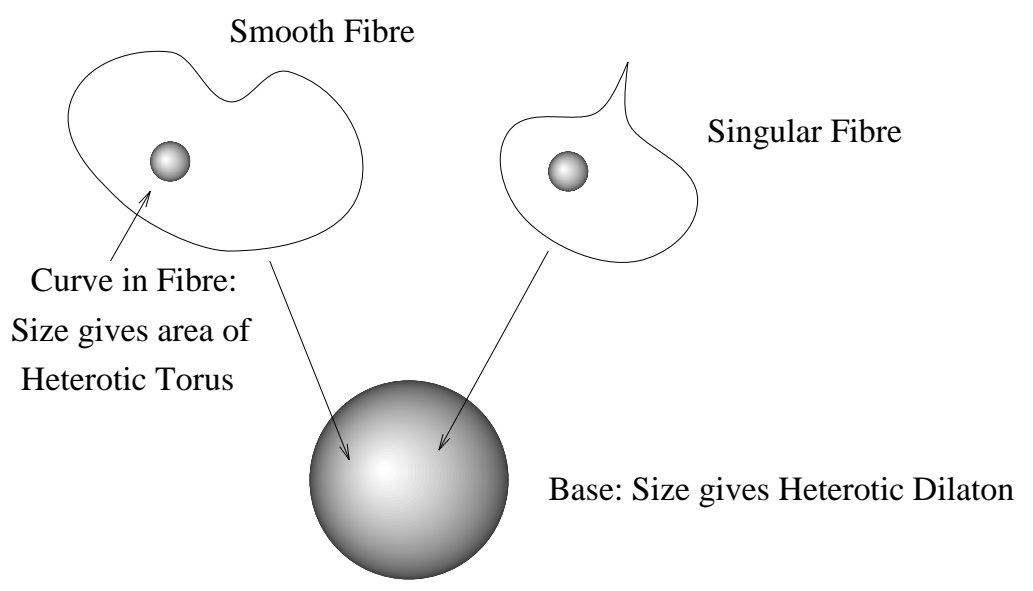

Figure 1: A K3 fibration with $h^{1,1}=3$.

Thus the first $\mathbb{P}^{1}$ can be seen as the base for writing the whole threefold as a K3 fibration. Clearly we could have chosen instead to fix a point first in the second $\mathbb{P}^{1}$ factor so $X$ admits two K3 fibrations.

We believe that the fact that there is only one K3 fibration for special values of the complex structure is probably not very significant from the point of view of string theory. It appears to be tied up with the phenomenon observed by Wilson [ 13] that the Kähler cone for a Calabi-Yau manifold can "jump" as one moves about the moduli space of complex structures as rational curves suddenly jump into existence. One may worry that this may have adverse effects on rational curve counts along the lines of [14] but it turns out that any such rational curve will give a zero contribution when the analysis of [15] is performed. Similarly we expect the dual heterotic string to be unaffected.

Let us clarify the interpretation of our construction of the fibration in terms of the heterotic string. Let us again fix a generic point in the first $\mathbb{P}^{1}$ and consider building a K3 surface as an elliptic fibration over the second $\mathbb{P}^{1}$. This elliptic fibration has one section. This section thus defines a rational curve embedded inside this K3 surface. The size of this rational curve is given by the size of second $\mathbb{P}^{1}$. Now we fibre the threefold as a K3 fibration over the first $\mathbb{P}^{1}$. The result is shown in figure 11. Thus the size of one of the $\mathbb{P}^{1}$ 's in the base $\mathbb{P}^{1} \times \mathbb{P}^{1}$ gives the size of the rational curve in the generic fibre and the other gives the size of the base of the K3 fibration. As explained in [7] the size of the base gives the value of the dilaton for the heterotic string. What is the size of the other $\mathbb{P}^{1}$ in terms of the heterotic string?

Let $H_{1}$ and $H_{2}$ be our two heterotic strings. In six dimensions let their string couplings (exponential of the dilaton) be given by $\lambda_{6, H_{1}}$ and $\lambda_{6, H_{2}}$. In [9] it was shown that

$$
\lambda_{6, H_{1}}=\frac{1}{\lambda_{6, H_{2}}} .
$$


Now let us compactify further down to four dimensions to give a dual pair of heterotic strings with coupling constants $\lambda_{4, H_{1}}$ and $\lambda_{4, H_{2}}$. To do this we compactify on a two-torus. The fourdimensional coupling depends on the six-dimensional coupling and the area of this two torus. This area differs according to which heterotic string measures it [9]. Let us denote the area of the torus $T_{1}$ or $T_{2}$ depending on whether it is measured by $H_{1}$ or $H_{2}$. We then have

$$
\begin{aligned}
& \lambda_{4, H_{1}}^{2}=\frac{\lambda_{6, H_{1}}^{2}}{T_{1}}=\frac{\lambda_{6, H_{1}}^{2}}{T_{2} \cdot \lambda_{6, H_{1}}^{2}}=\frac{1}{T_{2}} \\
& \lambda_{4, H_{2}}^{2}=\frac{1}{T_{1}}
\end{aligned}
$$

Thus the size of the other $\mathbb{P}^{1}$ in the type IIA picture is immediately apparent — it is the size of the torus in the heterotic string.

Note that the act of exchanging the fibrations with $X$ generically leads to a change in the complex structure of $X$. That is, there is a $\mathbb{Z}_{2}$ symmetry in the moduli space of complex structures together with Kähler forms but not on either alone. Translated into the heterotic string language this tells us that as we map from one heterotic string to its dual partner, there is a non-trivial map on the moduli space of hypermultiplets. This is exactly what was found in [9].

For some values of the moduli of the hypermultiplets the exchange of the base $\mathbb{P}^{1}$ 's will be a symmetry of $X$. Thus we have arrived at a manifest geometric realization of the duality between the two heterotic strings in terms of a symmetry of the space on which the equivalent type IIA string is compactified. Note that some evidence of the symmetry in the context of type IIB strings has previously been noted in [5].

It is interesting to note that we can now go some way to prove the conjecture made in [ [3] that a type IIA string compactified on $X$ is indeed dual to the heterotic string above. This is because one may show that $X$ is the only Calabi-Yau manifold which is an elliptic fibration over $\mathbb{P}^{1} \times \mathbb{P}^{1}$ which has the right Hodge numbers. To more precise, suppose the following statements hold.

1. The heterotic-heterotic duality in six dimensions proposed in [9] is correct.

2. This heterotic string compactified on a torus down to four dimensions is dual to a type IIA string compactified on some manifold $X_{1}$.

3. The weakly-coupled heterotic string can be understood in terms of $X_{1}$ near its large radius limit.

We can then state that $X_{1}$ is isomorphic to $X$.

Finally let us prove this uniqueness. This is a little technical and the reader may wish to skip to the next section. Suppose $Y$ is any Calabi-Yau manifold with two K3 fibrations 
and $h^{2,1}(Y)=243$. Then we claim that $Y$ is of the same type as the $X$ constructed above. Indeed, the two distinct maps $Y \rightarrow \mathbb{P}^{1}$ induces an elliptic fibration $f: Y \rightarrow \mathbb{P}^{1} \times \mathbb{P}^{1}$. If $f$ does not have a section, then there is an elliptic Calabi-Yau threefold $j: J(Y) \rightarrow \mathbb{P}^{1} \times \mathbb{P}^{1}$, the Jacobian of $Y$, which is fiber-wise isomorphic to $f$ except for possibly some isolated fibers, and such that $j$ has a section. See [ 12] for details. Then $j$ is birationally equivalent to a Weierstrass model over $\mathbb{P}^{1} \times \mathbb{P}^{1}$ as in the footnote above. Since $h^{1,2}(Y)=h^{1,2}(X)$ and each $J(Y)$ can arise from only a finite number of different $Y^{\prime}$ in the same deformation class of $Y$ (as follows from the theory of [12]), we see that we obtain a dominant map from the moduli space of $Y$ to the moduli space of $X$. However, again using the theory of [12], for general $X$, there is no elliptic fibration $Y \rightarrow \mathbb{P}^{1} \times \mathbb{P}^{1}$ without a section whose Jacobian is $X$. Thus $j: Y \rightarrow \mathbb{P}^{1} \times \mathbb{P}^{1}$ must already have a section, so $Y$ is deformation equivalent to $X$.

\section{Enhanced Gauge Groups}

It was discussed in [9] that going between the dual heterotic strings exchanges the rôles of the perturbative gauge group and the nonperturbative gauge group. We will now see that this is also in accord with the double fibration structure.

The construction of the heterotic string in question began with a theory with gauge group $E_{7} \times E_{7}$. Thus was then removed by picking a generic value for the bundle moduli. Thus we know that the $U(1)^{4}$ gauge group in our four-dimensional theory can be enhanced to at least $E_{7} \times E_{7} \times U(1)^{4}$ by tuning the hypermultiplet moduli suitably. When we are at such a point of enhanced symmetry, some vector moduli become massless and we can move into a new branch of the moduli space roughly along the lines of [16]. The new theory at a generic point in its moduli space will have $\operatorname{rank}\left(E_{7} \times E_{7}\right)=14$ more vector multiplets than the original and fewer hypermultiplets since we had to specialize a bundle with structure group $E_{8} \times E_{8}$ to one of $S U(2) \times S U(2)$. An $S U(2)$ bundle with $c_{2}=12$ has a moduli space of 84 real dimensions and so the number of hypermultiplets lost is $(448-84) * 2 / 4=182$. Thus if this new heterotic string is dual to a type IIA string on a Calabi-Yau manifold $X^{\prime}$, we have $h^{1,1}\left(X^{\prime}\right)=17$ and $h^{2,1}\left(X^{\prime}\right)=61$.

The appearance of enhanced gauge symmetries in the context of type II string compactifications has been discussed in [17, 18, 19]. As seen from this new heterotic string, the $E_{7} \times E_{7}$ gauge group appears by varying the vector moduli. For our purposes it will be the analysis of [18] which will be of most use. There it was claimed that the enhanced gauge groups which could be seen perturbatively by the heterotic string as the vector moduli varied must originate from singularities in the K3 fibre. Thus, the $E_{7} \times E_{7}$ gauge group appears when the generic K3 fibre has two $E_{7}$ singularities in it [2]. So long as this extremal transition between $X$ and $X^{\prime}$ preserves the K3 fibration structure then we can see that the $E_{7} \times E_{7}$ gauge group appears in the original heterotic string when we deform the complex structure of $X$ so as to acquire two $E_{7}$ singularities in the generic fibre.

We can do this explicitly as follows. Let us consider $X$ as an elliptic fibration over $\mathbb{P}^{1} \times \mathbb{P}^{1}$. 


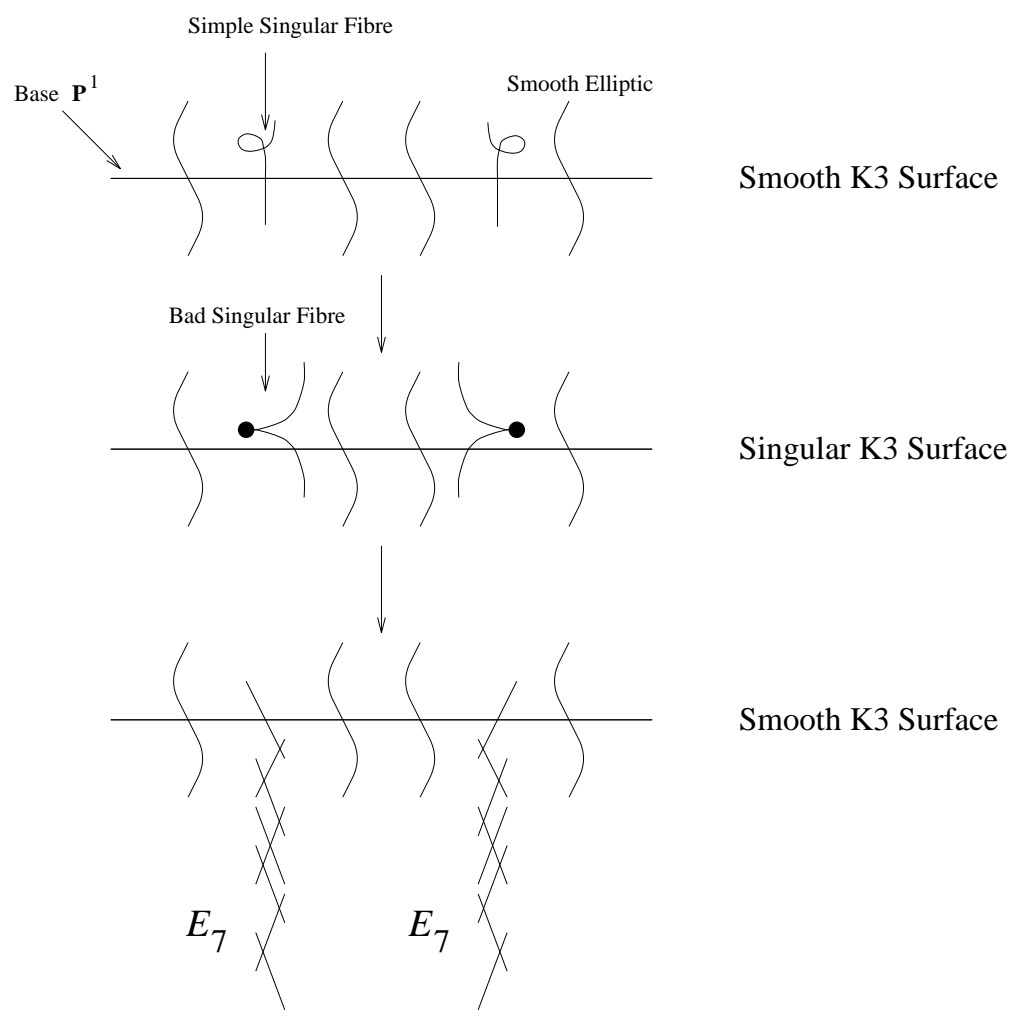

Figure 2: A transition of the generic K3 fibre.

When viewed as a K3 fibration, the generic fibre will itself be an elliptic fibration. Let us denote the homogeneous coordinates of the base $\mathbb{P}^{1} \times \mathbb{P}^{1}$ by $\left[s_{0}, s_{1}\right]$ and $\left[t_{0}, t_{1}\right]$. A fibre can then be written in Weierstrass form as

$$
y^{2}=x^{3}+a\left(s_{i}, t_{i}\right) x+b\left(s_{i}, t_{i}\right) .
$$

In order for the resulting threefold to be a Calabi-Yau manifold we require that $a$ is of bidegree $(8,8)$ in $s_{i}$ and $t_{i}$ and that $b$ be of bidegree $(12,12)$ [12].

As we move over the base $\mathbb{P}^{1} \times \mathbb{P}^{1}$, the discriminant of the elliptic, $4 a^{3}+27 b^{2}$, will have zeros leading to singular fibres. If the polynomials $a$ and $b$ are generic, the singular fibres will not be too bad and the resulting Calabi-Yau manifold will be smooth. For special choices of the polynomials however, the singular fibre will be sufficiently bad as to introduce singularities into the Calabi-Yau threefold. This has been discussed in the context of string theory and $D$-branes in [17].

Let us fix a generic point on one of the $\mathbb{P}^{1}$ 's (i.e., specify $t_{0}$ and $t_{1}$ ) in the base. The elliptic fibration given in (4) will then give the generic K3 fibre as an elliptic fibration. We now want to deform this as to get two $E_{7}$ singularities in the K3 surface. Following the work 
of [20] we can do this by choosing the following polynomials for $a$ and $b$ :

$$
\begin{aligned}
& a=s_{0}^{3} s_{1}^{3} f\left(s_{i}, t_{i}\right) \\
& b=s_{0}^{5} s_{1}^{5} g\left(s_{i}, t_{i}\right),
\end{aligned}
$$

where $f$ is of bidegree $(2,8)$ and $g$ is of bidegree $(2,12)$. We now claim that the generic K3 fibre has an $E_{7}$ singularity at $s_{0}=0$ and another at $s_{1}=0$. We can now blow each K3 fibre up to resolve this singularity.

This process of deforming the complex structure to acquire a singularity and then blowing it up is shown for a generic K3 fibre in figure 2. We show complex dimensions as real. In the each case the $\mathrm{K} 3$ surface is an elliptic fibration over the base $\mathbb{P}^{1}$. The blowing up procedure introduces two sets of rational curves in the shape of the Dynkin diagram for $E_{7}$.

In terms of the generic K3 fibre, this process has simply changed one K3 surface into another. The effect globally on the Calabi-Yau threefold is not so trivial however. We need to worry that the degenerate fibres of the K3 fibration may be sufficiently bad as to introduce singularities into the Calabi-Yau threefold. It may be shown that this is not the case here. Our new smooth Calabi-Yau manifold obtained by this extremal transition is, of course, the obvious candidate for $X^{\prime}$. Indeed one can show that it satisfies $h^{1,1}=17$ and $h^{2,1}=61$.

We claim then that we have constructed the Calabi-Yau manifold $X^{\prime}$ which is dual to the heterotic string obtained from the $E_{7} \times E_{7}$ heterotic string deformed by vector moduli to obtain a string with gauge group $U(1)^{4+14}$. Thus we have another example to add to those in [3, 21] of the geometric interpretation of a phase transition in the heterotic string in terms of the type IIA string.

Now let us ask what happens when we exchange the $\mathbb{P}^{1}$ 's in the base of the elliptic fibration as we try to go through this extremal transition. The base of the K3 fibration is now what was the base of generic K3 fibre when it was written as an elliptic fibration. Thus, we have our $E_{7}$ trees sticking out over a finite number of points on this base as shown in the bottom of figure 2. That is, this contribution to the counting of the vector multiplets comes from having reducible bad fibres in the K3 fibration. This is exactly the situation where we expect to have nonperturbative contributions to the gauge group of the heterotic string as explained in [ []. Thus we see that the $E_{7} \times E_{7}$ that was perturbative in the original heterotic string becomes nonperturbative in the dual heterotic string. What's more, one can also see that the generic fibre of the second fibration will not contain any singularities and so the perturbative gauge group consists only of the $U(1)^{4}$ which is always present.

It is easy to extend this analysis to the simply-laced gauge groups which are subgroups within $E_{7} \times E_{7}$ and in each case we see the structure conjectured in [9] appearing. That is, the parts of the gauge group which are perceived as perturbative and nonperturbative are exchanged (except for the $U(1)^{4}$ ) between the dual heterotic strings.

Up to this point we have managed to reproduce the effects seen in [9] in terms of the geometry of the type IIA string. It turns out that we can say more however. The 
same method as was used to introduce two $E_{7}$ singularities into the generic K3 fibre can actually be used to introduce two $E_{8}$ singularities. Thus we appear to be claiming that the heterotic string can be deformed to a theory with a gauge group containing $E_{8} \times E_{8}$. This is troublesome to interpret in terms of the usual picture since the centre of $E_{8} \times E_{8}$ is trivial — we need to deform our $E_{8} \times E_{8}$ bundle so that it becomes trivial and yet maintain $c_{2}=24$. Clearly this is impossible. What we can do however in addition is to bend the base K3 around and possibly introduce singularities into the base, or the bundle (perhaps reinterpreting it as a sheaf). (One may also try to analyze this in terms of open strings along the lines of [22].) Such processes can introduce further gauge groups. One should also suspect that this may introduce nonperturbative gauge groups however following Witten's work [23]. We will now see that this is indeed the case.

Something new happens when we degenerate to an $E_{8} \times E_{8}$ singularity compared to this cases of $E_{7} \times E_{7}$ and its subgroups. That is, when we blow-up to resolve the generic fibre it turns out that some singular fibres are still sufficiently badly-behaved as to make the resulting Calabi-Yau threefold singular. If we want to obtain a smooth threefold we require further blow-ups within these bad fibres. Thus we necessarily have contributions to $h^{1,1}$ of the threefold from bad fibres and thus, following [7], we have a nonperturbative part to the gauge group.

In the case at hand one can show that each of six bad fibres for each $E_{8}$ need to be blown up once to smooth the threefold. This suggests that we have a nonperturbative gauge group of rank 12 when we try to enhance the perturbative part of the gauge group to include $E_{8} \times E_{8}$. It is nice to see that a nonperturbative contribution to the gauge group arises when one tries to enhance to $E_{8} \times E_{8}$ but we have no definite geometric interpretation for this on the heterotic side yet in terms of singular bundles and/or singular K3 surfaces. We should say that finding the heterotic string description of this model is necessarily going to be difficult since there is always some nonperturbative contribution to the gauge group for any point in its moduli space.

\section{An Extreme Case}

We have discussed above an example of a Calabi-Yau manifold which admits two K3 fibrations. Actually there is no limit to the number of K3 fibrations one may obtain and in this section we discuss an example with an infinite number. In a way this will turn out to be more trivial than the previous case.

When one has more than one K3 fibration, the relationship between the different fibrations can be of one of three types:

1. The fibrations have quite different generic fibres. That is, the Picard lattice for the generic fibres differ. 
2. The generic fibres have the same Picard lattice but, for a generic complex structure, the act of exchanging the fibrations has a nontrivial action on the complex structure.

3. The fibrations are completely diffeomorphic.

An example of the first case is $X^{\prime}$ from section 3. After going through the $E_{7} \times E_{7}$ transition, the Picard lattice the generic K3 fibre will now contain the root lattice of $E_{7} \times E_{7}$ whereas the generic fibre of the other fibre will not. In terms of the heterotic string case 1 corresponds to the perturbative part of the gauge group for one heterotic string being different to the perturbative part for the other. In the case of $X^{\prime}$, one string has a large perturbative gauge group of rank 18 , whereas its dual partner only sees $U(1)^{4}$.

An example of the second case is $X$. Both heterotic strings have a perturbative gauge group of $U(1)^{4}$ but, as discussed in [9], the map between them is nontrivial on the hypermultiplet moduli space.

We will now discuss an example of the third type following [ 24]. Consider the CalabiYau manifold, $Y$, obtained by blowing up the orbifold $T^{6} /\left(\mathbb{Z}_{2} \times \mathbb{Z}_{2}\right)$. This orbifold has been discussed many times in the string literature, for example [25].

The first $\mathbb{Z}_{2}$ quotient may be thought of as building a K3 surface as $T^{4} / \mathbb{Z}_{2}$. The second quotient then acts on this K3 surface and the other $T^{2}$ to build a Calabi-Yau space. Let us analyze this intermediate K3 surface and in particular its Picard lattice, $\Gamma$ (see, for example [ 26] for a discussion of the Picard lattice in this context). The two $T^{2}$ 's used to build this K3 surface each contribute a rational curve class to the K3 surface as well as the 16 exceptional divisors coming from blowing up the orbifold. Thus, for a generic model, $\Gamma$ is a rank 18 lattice of signature $(1,17)$. Note that this lattice is not self-dual.

Now, thanks to the work of [27], it turns out that any primitive element of length 0 in $\Gamma$ defines the class of an elliptic curve in the K3 surface and that there is an elliptic fibration of the K3 surface whose generic fibre is this class. Thus, since there are an infinite number of such vectors, there are an infinite number of elliptic fibrations for such a K3 surface.

Now divide out by the second $\mathbb{Z}_{2}$. There are two possibilities for how this $\mathbb{Z}_{2}$ acts on our elliptic fibration of the K3 surface. It may either fix the base $\mathbb{P}^{1}$ point-wise or it may not. In the case that the base $\mathbb{P}^{1}$ is fixed, one may show that this may be used as the base of $Y$ as a K3 fibration [24]. Loosely speaking, this happens for "half" of the primitive elements of length zero in $\Gamma$. Thus $Y$ has an infinite number of K3 fibrations.

If our assertion in the introduction concerning the existence of a heterotic string for every K3 fibration is correct then we have an infinite number of heterotic strings any two of which are dual to each other!

Actually this is not as bad as it sounds. One can see that a subgroup (of finite index) of $O(1,17 ; \mathbb{Z})$ must act as a symmetry group on this Calabi-Yau manifold since it acted as such on the K3 surface above. This can be used to identify all the different K3 fibrations we have generated. Thus all the K3 fibrations are diffeomorphic. 
One should note that this does not mean that this example is trivial however. One should think of this subgroup of $O(1,17 ; \mathbb{Z})$ as acting as a group of $U$-dualities on the heterotic string in the sense of [1]. The group acts nontrivially on the dilaton and so identifies heterotic strings that do not look isomorphic from their conformal field theories. One should also note that it mixes contributions to $h^{1,1}$ from generic fibres and from bad fibres and so mixes perturbative gauge groups with nonperturbative gauge groups as in the earlier example. The difference between this example and that in section 2 is that this time the duality maps acts trivially on the space of hypermultiplets.

In [28] a conjecture was made about Calabi-Yau manifolds with such infinite symmetries. If it is correct then it will always be the case that a Calabi-Yau manifold with an infinite number of fibrations will lead to heterotic strings which are mainly equivalent in this manner. Dividing out by this group of dualities one can be left with only a finite number of heterotic strings which are equivalent in the sense of type 1 or type 2 above.

\section{Acknowledgements}

We would like to thank M. Duff, S. Kachru, V. Kaplunovsky and D. Morrison for useful conversations. The work of the authors is supported by grants from the National Science Foundation.

\section{References}

[1] C. Hull and P. Townsend, Unity of Superstring Dualities, Nucl. Phys. B438 (1995) 109-137.

[2] E. Witten, String Theory Dynamics in Various Dimensions, Nucl. Phys. B443 (1995) 85-126.

[3] S. Kachru and C. Vafa, Exact Results For N=2 Compactifications of Heterotic Strings, Nucl. Phys. B450 (1995) 69-89.

[4] S. Ferrara, J. Harvey, A. Strominger, and C. Vafa, Second Quantized Mirror Symmetry, Phys. Lett. 361B (1995) 59-65.

[5] A.Klemm, W.Lerche, and P.Mayr, K3-Fibrations and Heterotic-Type II String Duality, Phys. Lett. 357B (1995) 313-322.

[6] C. Vafa and E. Witten, Dual String Pairs With $N=1$ and $N=2$ Supersymmetry in Four Dimensions, Harvard and IAS 1995 preprint HUTP-95/A023, hep-th/9507050.

[7] P. S. Aspinwall and J. Louis, On the Ubiquity of K3 Fibrations in String Duality, Cornell and Munich 1995 preprint CLNS-95/1369, LMU-TPW 95-16, hep-th/9510234, to appear in Phys. Lett. B.

[8] D. R. Morrison, Mirror Symmetry and Rational Curves on Quintic Threefolds: A Guide For Mathematicians, J. Amer. Math. Soc. 6 (1993) 223-247. 
[9] M. J. Duff, R. Minasian, and E. Witten, Evidence for Heterotic/Heterotic Duality, Texas A\&M 1996 preprint CTP-TAMU-54/95, hep-th/9601036.

[10] D. R. Morrison and C. Vafa, Compactifications of F-Theory on Calabi-Yau Threefolds - I, to appear.

[11] N. Nakayama, On Weierstrass Models, in "Algebraic Geometry and Commutative Algebra in Honor of Masayoshi Nagata", pages 405-431, Kinokuniya, Tokyo, 1988.

[12] M. Gross, A Finiteness Theorem for Elliptic Calabi-Yau Threefolds, Duke Math. J. 74 (1994) 271-299.

[13] P. M. H. Wilson, The Kähler Cone on Calabi-Yau Threefolds, Invent. Math. 107 (1992) $561-583$.

[14] P. Candelas, X. C. de la Ossa, P. S. Green, and L. Parkes, A Pair of Calabi-Yau Manifolds as an Exactly Soluble Superconformal Theory, Nucl. Phys. B359 (1991) 21-74.

[15] P. S. Aspinwall and D. R. Morrison, Topological Field Theory and Rational Curves, Commun. Math. Phys. 151 (1993) 245-262.

[16] B. R. Greene, D. R. Morrison, and A. Strominger, Black Hole Condensation and the Unification of String Vacua, Nucl. Phys. B451 (1995) 109-120.

[17] M. Bershadsky, V. Sadov, and C. Vafa, D-Strings and D-Manifolds, Harvard 1995 preprint HUTP-95/A035, hep-th/9510225.

[18] P. S. Aspinwall, Enhanced Gauge Symmetries and Calabi-Yau Threefolds, Cornell 1995 preprint CLNS-95-1375, hep-th/9511171, to appear in Phys. Lett. B.

[19] S. Katz, D. R. Morrison, and M. R. Plesser, Enhanced Gauge Symmetry in Type II String Theory, Oklahoma et al 1996 preprint OSU-M-96-1, hep-th/9601108.

[20] R. Miranda, Smooth Models for Elliptic Threefolds, in R. Friedman and D. R. Morrison, editors, "The Birational Geometry of Degenerations", Birkhauser, 1983.

[21] P. S. Aspinwall, An $N=2$ Dual Pair and a Phase Transition, Cornell 1995 preprint CLNS95/1366, hep-th/9510142, to appear in Nucl. Phys. B.

[22] E. G. Gimon and J. Polchinski, Consistency Conditions for Orientifolds and D-Manifolds, ITP 1996 preprint NSF-ITP-96-01, hep-th/9601038.

[23] E. Witten, Small Instantons in String Theory, IAS 1995 preprint IASSNS-HEP-95-87, hepth/9511030.

[24] K. Oguiso, On Algebraic Fiber Space Structures on a Calabi-Yau 3-fold, Int. J. of Math. 4 (1993) 439-465.

[25] C. Vafa and E. Witten, On Orbifolds with Discrete Torsion, J. Geom. Phys. 15 (1995) 189-214. 
[26] W. Barth, C. Peters, and A. van de Ven, Compact Complex Surfaces, Springer, 1984.

[27] I. I. Piateckiü-Shapiro and I. R. Shafarevich, A Torelli Theorem for Algebraic Surfaces of Type K3, Math. USSR Izv. 5 (1971) 547-588.

[28] D. R. Morrison, Compactifications of Moduli Spaces Inspired by Mirror Symmetry, in A. Beauville et al., editors, "Journées de Géométrie Algébrique d'Orsay (Juillet 1992)", volume 218 of Astérisque, pages 243-271, Société Mathématique de France, 1993. 